\title{
Supplementary Information for: Understanding and Tracking the Excess Proton in Ab Initio Simulations; Insights from IR Spectra
}

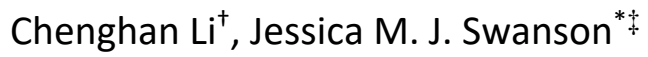

tDepartment of Chemistry, Chicago Center for Theoretical Chemistry, James Franck Institute, and Institute for Biophysical Dynamics, The University of Chicago, Chicago, Illinois 60637, United States

¥Department of Chemistry, Biological Chemistry Program, and Center for Cell and Genome Science, The University of Utah, Salt Lake City, Utah 84112, United States

\section{Proof of donor identity independency of rCEC}

In order to show rCEC is not dependent on the identity of donor atom, we first consider a twostate case (Figure S2). When state searching starts from atom $I$, following eq 6 and 8 ,

$$
\begin{gathered}
c_{i}^{2}=\frac{1}{1+c_{j}^{2} / c_{i}^{2}}=\frac{1}{1+f_{i j}^{\mathrm{ct}}} \\
c_{j}^{2}=c_{j}^{2} / c_{i}^{2} \times c_{i}^{2}=\frac{f_{i j}^{\mathrm{ct}}}{1+f_{i j}^{\mathrm{ct}}}
\end{gathered}
$$

While regarding $J$ as pivot, the coefficients are

$$
\begin{gathered}
c_{j}^{2}=\frac{1}{1+c_{i}^{2} / c_{j}^{2}}=\frac{1}{1+f_{j i}^{\mathrm{ct}}} \\
c_{i}^{2}=c_{i}^{2} / c_{j}^{2} \times c_{j}^{2}=\frac{f_{j i}^{\mathrm{ct}}}{1+f_{j i}^{\mathrm{ct}}}
\end{gathered}
$$

Recall that the charge transfer factor from state $|i\rangle$ to state $|j\rangle$ is $f_{i j}^{\text {ct }}=\exp \left(-k \delta_{I J H}\right)$ while $f_{j i}^{\text {ct }}=\exp \left(-k \delta_{J I H}\right)$. This leads to the fact that

$$
f_{i j}^{\mathrm{ct}} f_{j i}^{\mathrm{ct}}=1
$$

given that $\delta_{I J H}=r_{J H}-r_{I H}=-\delta_{J I H}$. Then, we have from eq S2 


$$
\begin{aligned}
& c_{j}^{2}=\frac{1}{1+f_{j i}^{\mathrm{ct}}}=\frac{f_{i j}^{\mathrm{ct}}}{f_{i j}^{\mathrm{ct}}+f_{i j}^{\mathrm{ct}} f_{j i}^{\mathrm{ct}}}=\frac{f_{i j}^{\mathrm{ct}}}{f_{i j}^{\mathrm{ct}}+1} \\
& c_{i}^{2}=\frac{f_{j i}^{\mathrm{ct}}}{1+f_{j i}^{\mathrm{ct}}}=\frac{f_{i j}^{\mathrm{ct}} f_{j i}^{\mathrm{ct}}}{f_{i j}^{\mathrm{ct}}+f_{i j}^{\mathrm{ct}} f_{j i}^{\mathrm{ct}}}=\frac{1}{f_{i j}^{\mathrm{ct}}+1}
\end{aligned}
$$

Thus, the coefficients starting from I (eq S1) are recovered, i.e. rCEC is invariant with donor identity change in the two-state case. The conclusion can be easily generalized to the multistate by noticing the coefficient of any state $\left|i_{k}\right\rangle$ is propagated from a parent state $\left|i_{1}\right\rangle$ by multiplying $f_{\mathrm{ct}}$ 's along a hydrogen bond chain $\left|i_{1}\right\rangle \rightarrow\left|i_{2}\right\rangle \rightarrow \cdots \rightarrow\left|i_{k}\right\rangle$. An alternative donor index may change $\left|i_{k}\right\rangle$ into the parent of $\left|i_{1}\right\rangle$. Thus, the fact that $f_{i_{k} i_{1}}^{\mathrm{ct}} f_{i_{1} i_{k}}^{\mathrm{ct}}=f_{i_{2} i_{1}}^{\mathrm{ct}} f_{i_{1} i_{2}}^{\mathrm{ct}}$. $f_{i_{3} i_{2}}^{\mathrm{ct}} f_{i_{2} i_{3}}^{\mathrm{ct}} \cdots f_{i_{k} i_{k-1}}^{\mathrm{ct}} f_{i_{k-1} i_{k}}^{\mathrm{ct}}=1$ means the relative ratio of $c_{i_{1}}^{2}$ and $c_{i_{k}}^{2}$ is independent of the donor index. Additionally, $\left\{c_{i}^{2}\right\}$ 's are normalized to 1 , so identical $c_{j}^{2} / c_{i}^{2}$ for $\forall i, j$ results in identical $\left\{c_{i}^{2}\right\}$.

\section{Vibrational intensity of $c_{i}^{2}$}

We also computed the vibrational intensity (vibrational density of states; VDOS) of the state population $c_{i}^{2}$ (Figure S3A) from

$$
\operatorname{VDOS}_{i}(\omega)=\int\left\langle c_{i}^{2}(0) c_{i}^{2}(t)\right\rangle e^{-i \omega t} \mathrm{~d} t
$$

Noticeably, the $2800 \mathrm{~cm}^{-1}$ frequency can be seen in the fourth largest $c_{i}^{2}$, which includes the contributions from proton stretch modes of $\mathrm{O}_{0}-\mathrm{O}_{1 z}$ and also $\mathrm{O}_{0}-\mathrm{O}_{1 \mathrm{y}}, \mathrm{O}_{1 \mathrm{x}}-\mathrm{O}_{2 \times 1}$ and $\mathrm{O}_{1 \mathrm{x}}-\mathrm{O}_{2 \times 2}$ in a more Zundel-like configuration (Figure S3B). Noting that $\mathrm{O}_{2 \times 1}$ and $\mathrm{O}_{2 \times 2}$ are the second solvation shell of the $\mathrm{H}_{3} \mathrm{O}$ core, $2800 \mathrm{~cm}^{-1}$ frequency of the $2^{\text {nd }}$ shell in Figure $1 \mathrm{C}$ could be attributed to these proton stretch modes. This additionally verifies the rCEC is capturing the vibrational modes in the hydrated proton complex but the decaying excess charges hide the IR signal in CEC's spectrum. 


\section{SI Figures}

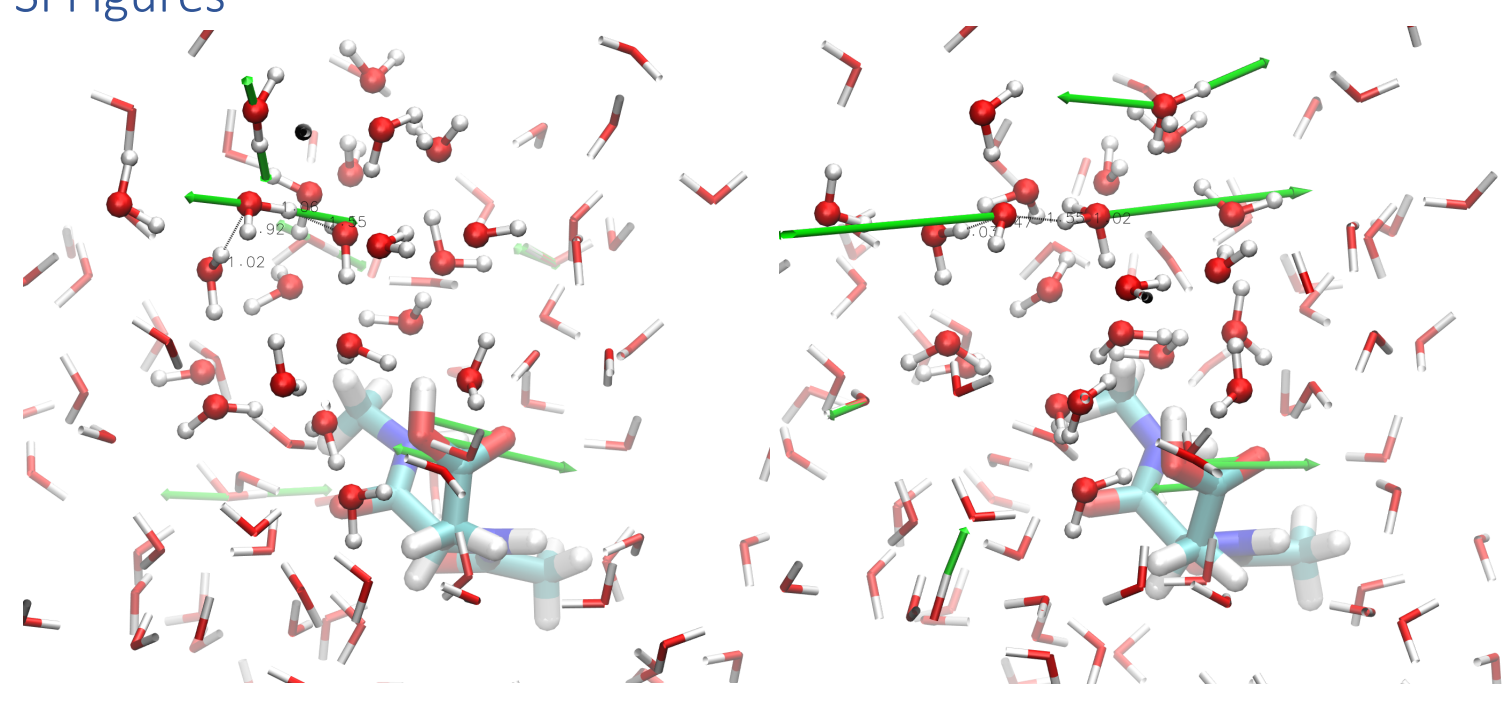

Figure S1. Snapshots extracted from $5527.5 \mathrm{fs}$ and $6136.5 \mathrm{fs}$ of the metadynamics simulation of aspartic acid in water showing the starting point and the resulting configuration of the decomposition procedure. The bias forces acting on atoms are shown by green arrows with a scale of $25 \mathrm{~kJ} / \mathrm{mol} / \AA^{2}$ force per $1 \AA$ arrow length. Forces under $25 \mathrm{~kJ} / \mathrm{mol} / \AA^{2}$ are not shown for clarity.<smiles>OC(O)C(O)C(O)C1OC(O)C(O)C1O</smiles>

Figure S2. An illustration of two state case. The proton donor oxygen is denoted as the green ball and the red ball is denoting its acceptor.
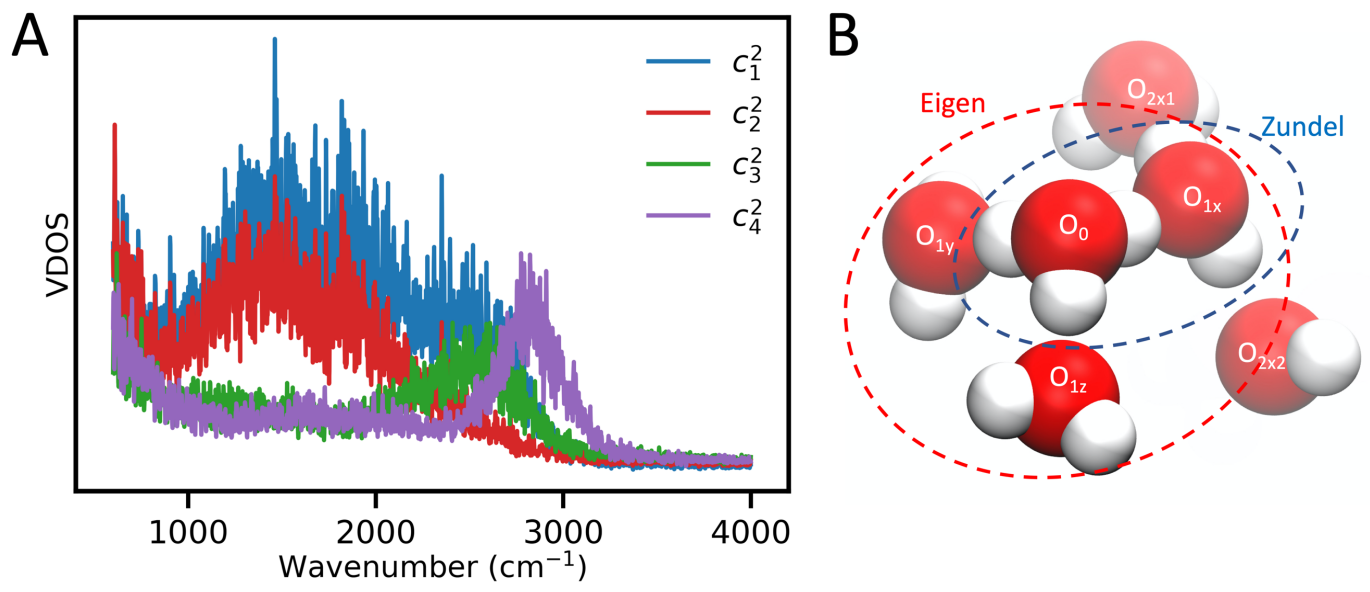
Figure S3. (A) The VDOS of the 4 largest $c_{i}^{2}$. (B) The oxygen notation. The oxygens with two largest $c_{i}^{2}$ are denoted as $\mathrm{O}_{0}$ and $\mathrm{O}_{1 \mathrm{x}}$. The other two oxygens in the first solvation shell of $\mathrm{O}_{0}$ are denoted as $\mathrm{O}_{1 y}$ and $\mathrm{O}_{1 z}$, in order of increasing $\delta$. The two closest oxygens in the first solvation shell of $\mathrm{O}_{1 \times}$ are denoted as $\mathrm{O}_{2 \times 1}$ and $\mathrm{O}_{2 \times 2}$.

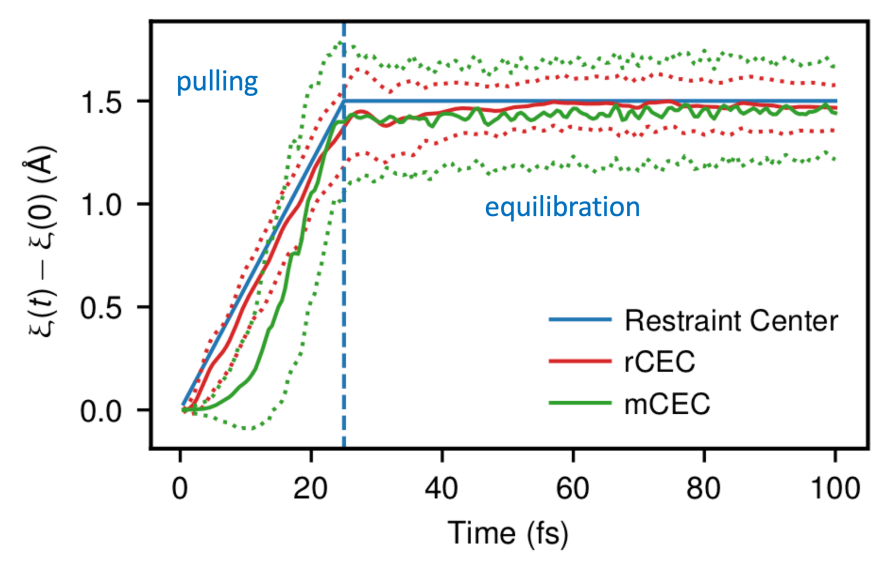

Figure S4. Time evolution of the deprotonation collective variable $\xi$ using rCEC (red) and mCEC (green) and the restraint center (blue) in SMD runs. The average value over all SMD runs (solid line) of rCEC follows the restraint center (blue) while mCEC shows an obvious hysteresis. The standard deviation interval (dotted lines) of rCEC is much smaller than mCEC. This suggests that, when pulling the $\mathrm{mCEC}$, the proton seems stickier to the amino acid, implying $\mathrm{mCEC}$ is missing some slow motions in the collective proton disassociation process, which may cause an overestimation of free energy barrier due to finite sampling. Also, the new CEC shows a much smaller standard deviation, revealing its robustness when handling various initial configurations. 